\title{
Study on RFID-Enabled Real-Time Vehicle Management System in Logistics
}

\author{
Yizhong Wang \\ School of Electronic Information and Automation \\ Tianjin University of Science and Technology \\ Tianjin, 300222, P. R. China \\ yzwang@tust.edu.cn
}

\author{
Oscar K.W. Ho and George Q. Huang \\ Department of Industrial and Manufacturing Systems \\ Engineering \\ The University of Hong Kong \\ Pokfulam Road, Hong Kong SAR, P. R. China \\ \{oscarhkw \& gqhuang\}@hkucc.hku.hk
}

\author{
Da Li and Huafang Huang \\ School of Electronic Information and Automation \\ Tianjin University of Science and Technology \\ Tianjin, 300222, P. R. China \\ \{lida \& hfhuang\}@tust.edu.cn
}

\begin{abstract}
In this paper, a real-time vehicle management system was proposed to plan and schedule vehicles' optimum routings using real-time information in logistics or distribution services. Three technologies were adopted for monitoring realtime situation and providing visualized interface, including radio frequency identification, geographical information system, and global positioning system. A real-time vehicle routing planning with time windows model and an insertion heuristic method were used to formulate vehicles' scheduling and search the relative optimal solution, respectively. The system was implemented by means of Visual Basic .Net and Visual Earth. Numerical experiments were conducted with good results to show the feasibility of the system.
\end{abstract}

Index Terms - RFID. Real time vehicle routing problem. Logistics. GIS. GPS.

\section{INTRODUCTION}

Today, the main pressure of many enterprises is how to reduce operational costs in order to create their competitive advantages that sustain their business in the global market. In tradition, the methods for reducing operational costs, such as mass production, total quality management, and inventory management, were often focused on the manufacturing area. However, the chance for further minimizing the manufacturing costs trends to very little in today manufacturing industries, such that many enterprises have been turning their focus on logistics management in order to reduce transportation costs. Effectively managing transportation system in logistics will not only enhance the performance in entire logistics processes but also reduce the transportation costs significantly.

Vehicle plays one of important roles in the transportation of logistics [1]. Optimized vehicle routing planning and scheduling produces substantial saving transportation process

This research is supported by a grant from Tianjin Municipal Science and Technology Commission under the code 08ZCKFNC00700.

978-1-4244-2503-7/08/\$20.00 C 2008 IEEE by minimizing the operational costs of transportation in logistics. The vehicle routing planning (VPR) is a well known topic that study on how to optimize costs by finding a set of tours of minimum length for a fleet of vehicles that initially is located at the depot then each vehicle follows its planned tour to delivery goods or services to customers such that customers' demands are satisfied and vehicles are fully utilized [2-3]. Taking into consideration the time windows of customers and depot with the real-time information of vehicles, customers, and depot extends VPR into real-time vehicle routing planning with time windows (real-time VRPTW) [4].

Nowadays, real-time technologies, such as radio frequency identification (RFID), geographical information system (GIS), and global positioning system (GPS), allow that a vehicle management system easily allocates resources, monitor processes, and intelligent manage information in order to reduce transportation costs while satisfying different customers' requirements.

In this paper, a real-time vehicle management system was proposed and implemented with the use of RFID, GIS, and GPS to monitor real-time situation and to provide visualized interface. With provided real-time information, the system formulates vehicles' scheduling and searches the relative optimal solution using a real-time VRPTW model and an insertion heuristic method. A three-tier client-server system was adopted as the architecture of the vehicle management system. The proposed system was programmed by using Visual Basic .Net and Visual Earth. Numerical experiments results showed the feasibility of the system.

\section{RFID, GPS, AND GIS}

Radio Frequency Identification is abbreviated as RFID and it is one of the automatic identification technologies used to identify an object or a person [5]. It consists of three main components, which are RFID tags, readers/writers, and its management system. The data communication of RFID between a tag and a reader/writer is achieved by using radio 
frequency and hence it can communicate without any contact. Generally speaking, memory capacity of RFID tags can store data up to $64 \mathrm{~K}$ [6]. It is much higher than other identification systems, such as bar-code

RFID technology does not require direct contact for finishing the data retrieval and processing. Also, it can work in different environments, even in the harsh environment. In the data security, RFID can apply some encryption algorithm, such as DES, RSA, DSA, and MD5, to provide security and privacy means for data communication [7]. Therefore, it has been widely using in manufacturing, supply chain, traffic control, distribution, medicine, tracking, and asset management to data collection and processing.

GPS is a satellite-based navigation system comprised of a network of satellites placed into orbit. It has a variety of application on land, at seam and in the air [8].

GIS can become a part of the decision support systems. It not only consists of functions in typical information systems but also it has ability to process those geographical data [9]. Therefore, it supports the functions of spatial searching and analysis. Currently, WebGIS, a type of GIS that provides services on the Web, has been adopted by many business firms to support their decision making [10] and GIS technology itself becomes a part of the general model in business field.

\section{VEHICLE MANAGEMENT SYSTEM}

\section{A. System Structure}

As shown in Fig. 1, the system of vehicle management comprised of GIS-GPS, RFID system, and a vehicle management information system.

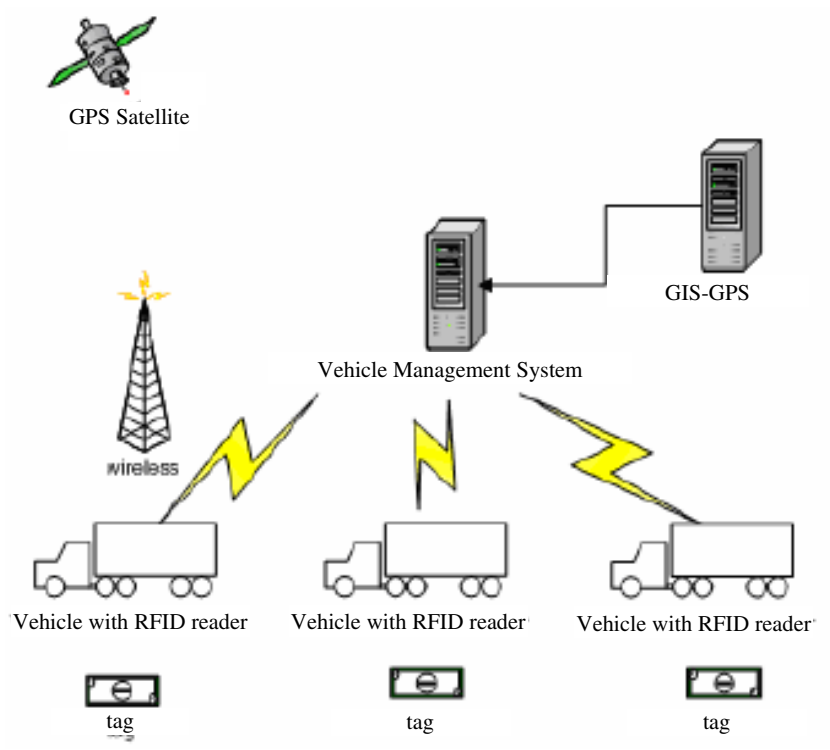

Fig. 1 Vehicle management system structure

GIS was used to provide spatial analysis such as searching the quickest path between two locations. Moreover, it provided information such as traffic condition or travel time between two locations such that the vehicle routing system planned the route for each vehicle based on those information.
GPS was used to positioning the locations of vehicles. RFID system, in vehicle management system, was used to update real-time status, such as arrival time or departure time of depots or destinations, of each vehicle to the database through its middleware and client system. The management information system was used to manage order information, such as changing, adding, or canceling orders. Also there were lots of functions, such as inventory control in its information system. However, in order to simplify the complexity of the vehicle management systems, those functions had been ignored in this paper.

\section{B. System Task Analysis}

The system was designed to serve two groups of users who were vehicle management system administrator and vehicle drivers. Fig. 2 showed the context level data flow diagram for the system and the information flow between management staff and drivers.

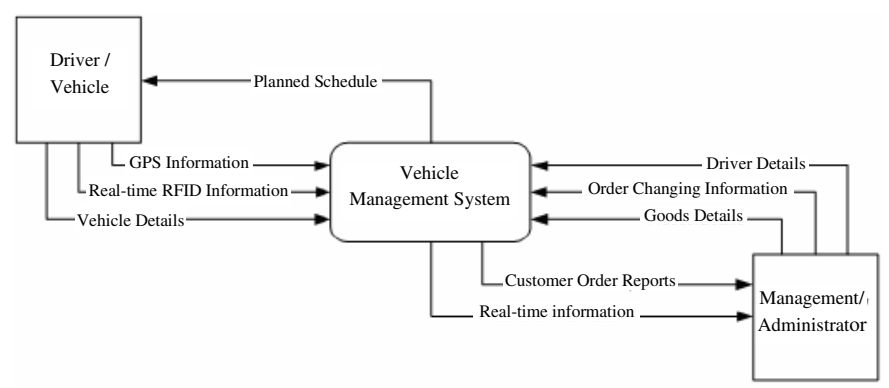

Fig. 2 System data flow diagram

Administrators had the responsibility to management the whole system and therefore they had the highest authority in the system. Generally, Administrators set the basic information to the system, such as company details, vehicle information, customer basic information, and information of goods to be delivered. Furthermore, he/she could change, add, cancel, and retrieve order information in order to prepare for scheduling. In addition to order management, administrators also performed the pre-schedule function to plan route for each vehicle in order to check whether it was feasible. Track and trace was another duty for an administrator or management staff that they monitored the real-time status of vehicles in order to make the real-time decision for delivery activities.

Vehicle drivers' tasks were very simple. First they obtained the information of planned route from the database. And then they followed the plan to carry out their delivery mission. Besides, drivers obtained the optimal path for delivery mission from GIS server.

Customers, of cause, were a major role of the system. They placed orders and managed their own orders in the system. However, in this paper, the focus was on the administrators and drivers. 


\section{System Architecture}

The architecture of the vehicle management system was the three-tier client-server system [11]. It was a one kind of computer called as clients initiating the process, while the other computers in the network called as servers were effectively slaves. The advantage of adopting this architecture is that it provides flexible processing in the distributed systems with better controlling in data management. The client-server system was developed by using .Net technology called .Net Remoting which is an updated version of DCOM. The whole picture of .Net Remoting was shown in Fig. 3.

In this model, whenever a client activated a remote object, two proxy objects were created in the client. A proxy object was a substitute for the remote object in the client side. It had the same methods and properties as the remote object but it didn't actually do anything in the client computer. The real work was being done by the server. These two proxies were also called as transparent proxy and the real proxy, respectively. Transparent proxy intercepted all the method, called directed to remote object, and passes the required values to real proxy. The real proxy was to handle all outbound communication and passed the message on to the channel sink chain. A sink means an element of software that can receive something. This chain of sinks carried out further processing on the method calls. The first sink in the chain carried out the formatting according to mechanism that had been registered. The final sink in the chain was the transport sink that was to send the byte stream out.

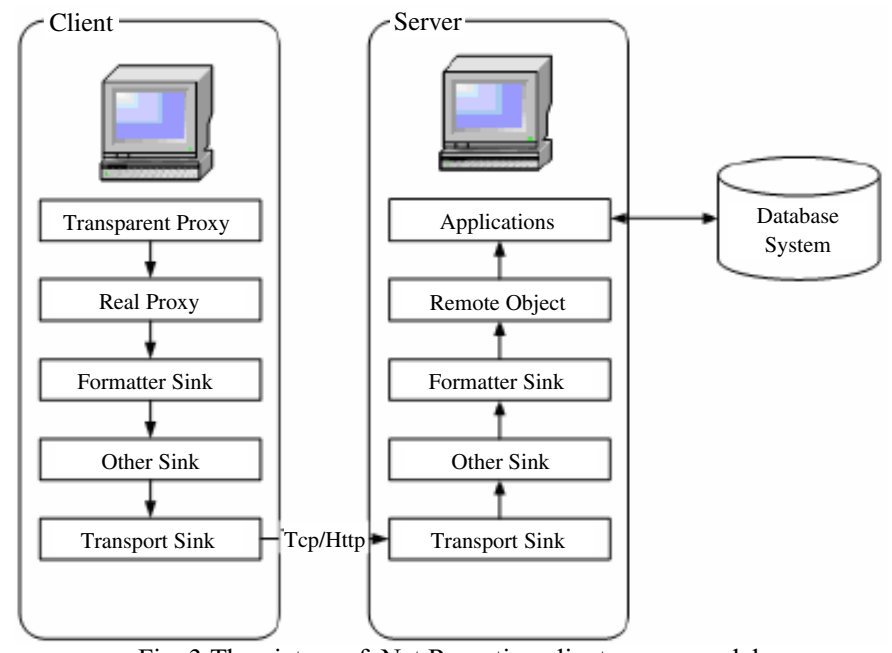

Fig. 3 The picture of .Net Remoting client-server model

At the server side, the transport sink received the incoming stream and passed it back trough the sink chain until it reached the formatter. The message was then deserialized and passed in to the remote object. The remote object implemented applications and gave the feedbacks to the client.

\section{Real-time VRPTW}

A real-time VRPTW model and a heuristic algorithm developed by Chen [12] was adopted and modified in this paper. Details of them can be got in Ref. [12]. Below was a brief description.
Whenever the real-time orders had been made or timedependent travel time had been changed, the real-time VRPTW model was necessary to schedule the routes again in order to optimize the travel time and fulfill all demands. Therefore, at the particular time, the real-time was formulated as a mixed integer problem, as follows:

Minimize:

$$
\sum_{i \in N u 0(\tau)} \sum_{j \in N u 0(\tau)} \sum_{k} c_{i j} x_{i j k}
$$

Subject to the following constraints: flow conservation constraints; time window and departure time constraints; vehicle capacity constraints; and definitional constraints.

For the VRPTW, the insertion method has been proven effective in constructing routes [12]. Therefore, a modified insertion method was adopted for route construction, as follow:

Step1. Input data

Step2. Classify customers and calculate earliest departure times

Step 3. Find the optimal place and departure time for each unassigned node and calculate its accrued cost

Step 4. Insert the customer and update the relevant data

Step 5. Stopping check for assignment

\section{E. Network Communication between Clients and the Server}

As above mentioned, the three-tier system and .Net remote object technology had been adopted to deploy the vehicle management system. However, the major difficult was that how to connect those distributed client computer with the system server in the depot. To overcome this difficult, the WiFi wireless network had been proposed for connecting between client computers in the truck and the server computer in the depots. Therefore, they used TCP protocol to communicate with each other wirelessly assumed that WiFi access points could cover all Hong Kong regions. Since WiFi technology will be further developed by Intel Corp. to become WiMax network, such improvement would enhance the system feasibility in the future.

\section{F. System Functions}

The structure of main system interface and the main functions of the proposed system were illustrated in Fig. 4 and Fig. 5, respectively.

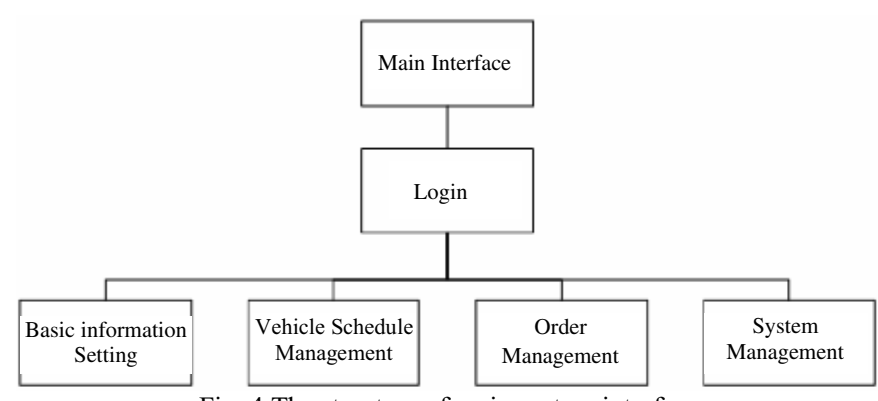

Fig. 4 The structure of main system interface 


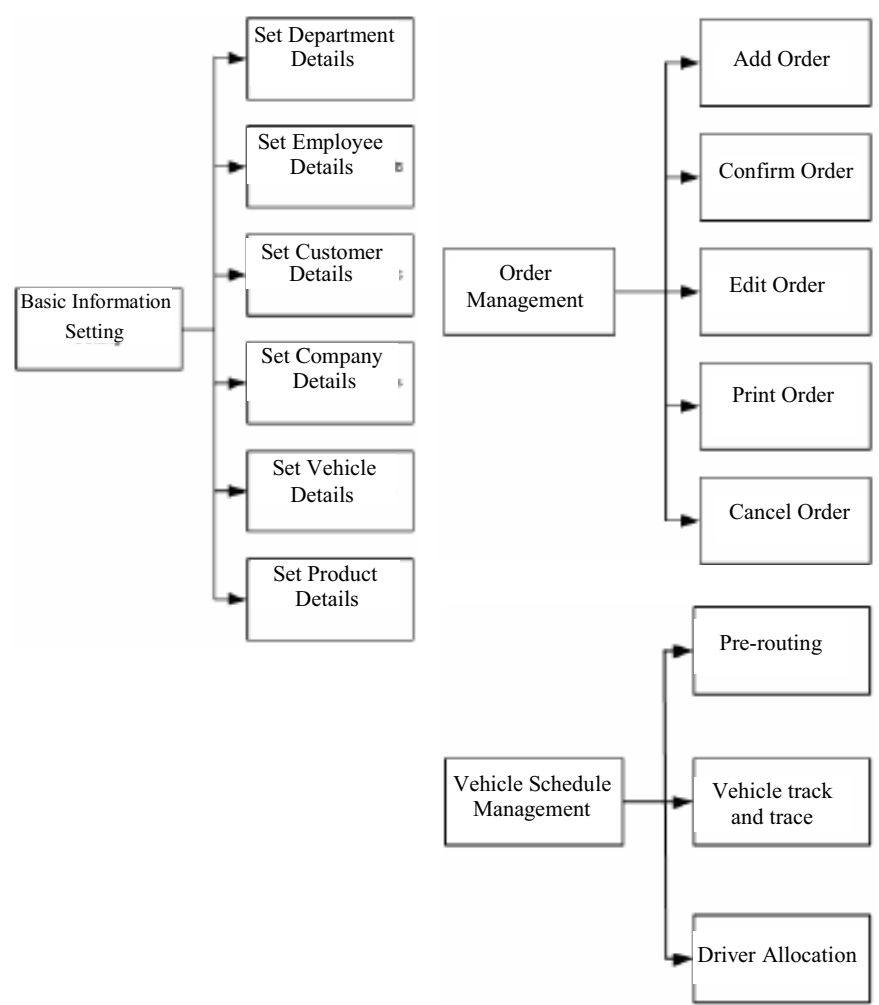

Fig. 5 The main functions of the system

\section{G. System Implementation}

Both client and server applications were developed in the Microsoft Visual Studio 2003. Visual Basic .Net was used as programming language. In order to achieve user-friendly interface, all most every window was added a searching function. The searching results were shown into a datagrid component. In addition, different information was shown with different colors. For example, as shown in Fig. 6, different colors in the data-grid component of vehicle tracking window indicated the different statuses of orders.

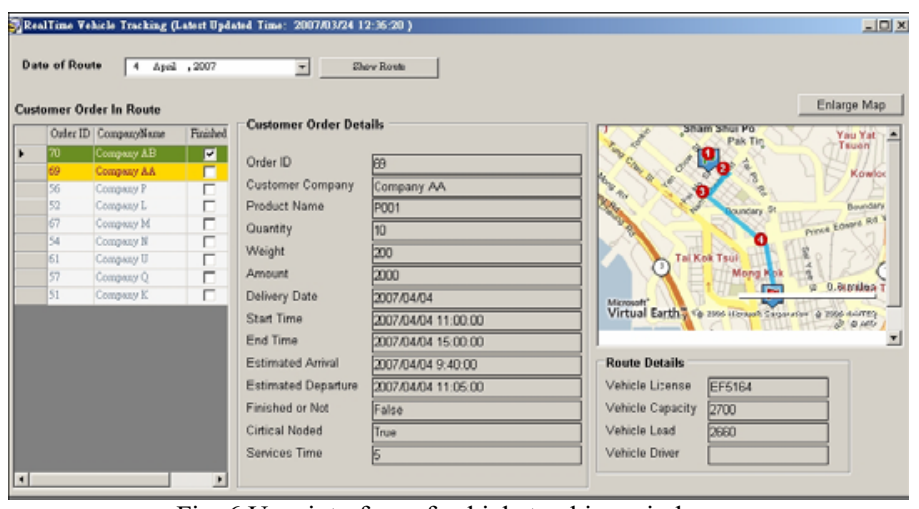

Fig. 6 User interface of vehicle tracking window

In this paper, Microsoft Virtual Earth Technology was adopted to develop a simplified GIS in the web server. The function of web GIS was used to search a quickest path between two locations and show position of a particular vehicle. Fig. 7 gave an example of showing the quickest path between tow locations.

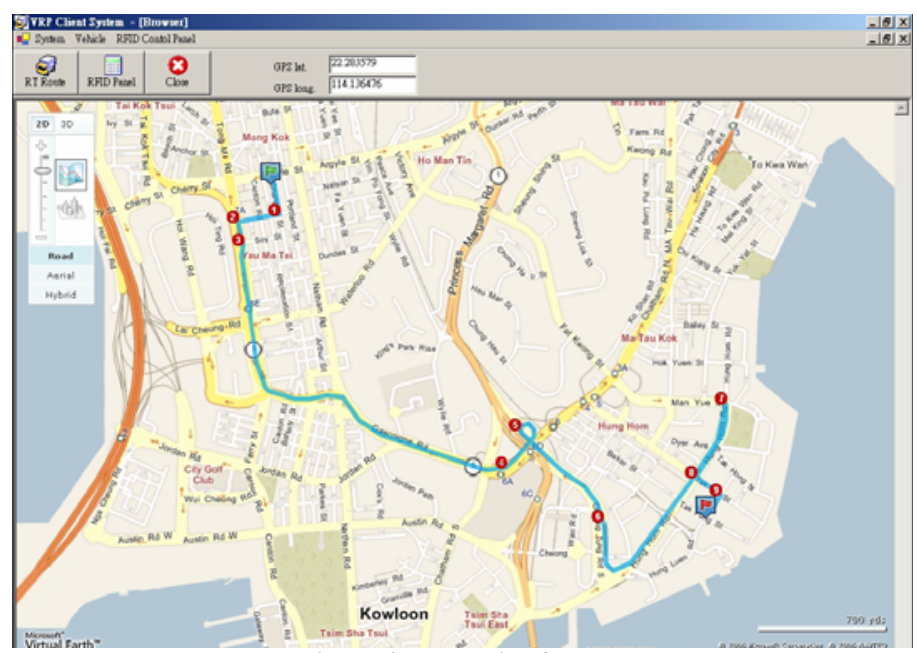

Fig. 7 The example of GIS

\section{NUMERICAL EXPERIMENTS AND RESULTS}

In order to test the proposed system, numerical experiments were conducted. Thirty customers who were dispersed in different locations in Hong Kong and three vehicles were created. The vehicles initially stayed at the depot of a company, which was assumed as a market leader to supply frozen foodstuffs in Hong Kong region. Then, thirty customers' orders were generated, which had different time windows for delivery. The summary of three performed tests was tabulated in Table 1.

The first 26 orders were initially used to construct the vehicle routes. In the first optimization test, there was no rejected customer order existing, and the schedule of each route was reasonable.

Then, after the time dependent departure time was changed, the routes were rescheduled in order to see the effects from the time dependent travel time function. The travel time between two locations was increased. Therefore, the total travel time was increased comparing with the first optimization test. The schedule of routes also was changed because of fulfilling the time windows constraints. Also, there was still no rejected order existing.

Finally, the last two orders were added to the schedule list then the schedule for each route was generated. Comparing the first test, the schedule of the final test was significant different from the first test, but it was still a reasonable schedule. Also the estimate arrival time for each company was increased due to new orders had inserted to routes and hence the duration of routes was longer than the first test. 
TABLE I

SUMMARY OF THE THREE OPTIMIZATION

\begin{tabular}{|c|c|c|c|c|}
\hline & & $1^{\text {st }}$ optimization & $2^{\text {nd }}$ optimization & $3^{\text {rd }}$ optimization \\
\hline \multirow{3}{*}{$\begin{array}{c}\text { Route No: } 1 \text { for the Vehicle } \\
\text { No. } 1\end{array}$} & Loading & $2660 \mathrm{~kg}$ & $2660 \mathrm{~kg}$ & $2700 \mathrm{~kg}$ \\
\hline & Order ID with sequence & $\begin{array}{l}56,52,67,54,62,61,60,59, \\
58,57,51\end{array}$ & $\begin{array}{l}51,57,58,59,60,61,62,54, \\
67,52,56\end{array}$ & $69,64,63,70,56,67,57,51$ \\
\hline & Est. Arrival Time & From 09:24 to $13: 01$ & From 09:31 to $14: 20$ & From 09:34 to $13: 27$ \\
\hline \multirow{3}{*}{$\begin{array}{l}\text { Route No: } 2 \text { for the Vehicle } \\
\text { No. } 2\end{array}$} & Loading & $2700 \mathrm{~kg}$ & $2700 \mathrm{~kg}$ & $2660 \mathrm{~kg}$ \\
\hline & Order ID with sequence & $\begin{array}{l}42,43,55,41,66,45,44,64, \\
63\end{array}$ & $\begin{array}{l}63,64,44,45,66,41,55,42, \\
43\end{array}$ & $\begin{array}{l}59,58,60,62,61,54,52,44, \\
45,66,41\end{array}$ \\
\hline & Est. Arrival Time & From 09:44 to $12: 27$ & From $10: 05$ to $14: 20$ & From 09:34 to $17: 21$ \\
\hline \multirow{3}{*}{$\begin{array}{l}\text { Route No: } 3 \text { for the Vehicle } \\
\text { No. } 3\end{array}$} & Loading & $1860 \mathrm{~kg}$ & $1860 \mathrm{~kg}$ & $2260 \mathrm{~kg}$ \\
\hline & Order ID with sequence & $68,48,47,46,65,40$ & $40,46,65,48,47,68$ & $\begin{array}{l}40,43,42,68,48,47,46,65, \\
55\end{array}$ \\
\hline & Est. Arrival Time & From 09:48 to $13: 32$ & From $10: 25$ to $15: 36$ & From $10: 25$ to $18: 58$ \\
\hline \multicolumn{2}{|c|}{ Rejected order } & Null & Null & Null \\
\hline
\end{tabular}

\section{CONCLUSION}

In this paper, a simplified vehicle management system was developed in order to demonstrate the idea for real-time vehicle management with using RFID, GPS, and GIS. In the system design, basing on the layout of the system, the system task analysis, the system architecture, the real-time VRPTW model, and the system functions were identified for implementing the system. VB .Net was used as a major programming language for system implementation. In order to test the proposed system, numerical experiments were conducted with good results. It is no doubt that implementing effective and efficiency vehicle management system will reduce operational costs significantly such that enterprises will maintain their competitive advantages to sustain their business.

\section{REFERENCES}

[1] T. Van Woensel, L. Kerbache, H. Peremans, and N. Vandaele, Vehicle routing with dynamic travel times: A queueing approach, European Journal of Operational Research, vol. 186, n. 3, pp. 990-1007, May 2008.

[2] R. Tavakkoli-Moghaddam, N. Safaei, and Y. Gholipour, A hybrid simulated annealing for capacitated vehicle routing problems with the independent route length, Applied Mathematics and Computation (New York), vol. 176, no. 2, pp. 445-454, May 2006.

[3] P. Toth and D. Vigo, The Vehicle Routing Problem, SIAM, 2002.

[4] K. Zhu and K Ong, A reactive method for real time dynamic vehicle routing problem, Proceedings of Twelfth Internationals Conference on Tools with Artificial Intelligence, Vancouver, BC, Canada, 13-15 November 2000, pp. 176-180.

[5] K. Finkenzeller, RFID-Handbook: Fundamentals and Applications in Contactless Smart Cards and Identification, Wiley \& Sons Ltd, 2003.

[6] http://www.morerfid.com/details.php?subdetail=Report\&action=details\&r eport $\mathrm{id}=2706 \&$ display $=$ RFID

[7] S. Garfinkel, RFID : Applications, Security, and Privacy, AddisonWesley, 2006.

[8] http://www.webopedia.com/TERM/G/GPS.htm

[9] H. Miller, Geographical Information Systems for Transportation: Principles and Applications, Oxford University Press, 2001.

[10]B. Yao, H. Lu, W. Shi, and X. Tong, A WebGIS based general framework of information management and aided decision making system for earthquake disaster reduction, Proceedings of 2006 IEEE International Geoscience and Remote Sensing Symposium, pp. 1568-1571, 2006.

[11]N. Depledge, W. Turner, and A. Woog, An open, distributable, three-tier client-server architecture with transaction semantics, Digital Technical Journal, vol. 7, no. 1, pp. 34-42, 1995.
[12]H. Chen, C. Hsueh, and M Chang, The real-time time-dependent vehicle routing problem, Transportation Research Part E, vol. 42, pp. 383-408, 2006. 